\title{
Brain functional abnormality in schizo-affective disorder: an fMRI study
}

\author{
M. Madre ${ }^{1,2,3}$, E. Pomarol-Clotet ${ }^{1,4}$, P. McKenna ${ }^{1,2,4}$, J. Radua ${ }^{1,4}$, J. Ortiz-Gil ${ }^{1,4,5}$, F. Panicali ${ }^{1,2,5}$, \\ J. M. Goikolea ${ }^{4,6}$, E. Vieta ${ }^{4,6}$, S. Sarró ${ }^{1,4}$, R. Salvador ${ }^{1,4}$ and B. L. Amann ${ }^{1,2,4 *}$ \\ ${ }^{1}$ FIDMAG Germanes Hospitalàries, Spain \\ 2 Benito Menni, CSMA, Barcelona, Spain \\ ${ }^{3}$ Departament de Psiquiatria i Medicina Legal, Doctorat de Psiquiatria i Psicologia Clínica, Universitat Autònoma de Barcelona, Spain \\ ${ }^{4}$ CIBERSAM, Spain \\ ${ }^{5}$ Hospital General de Granollers, Spain \\ ${ }^{6}$ Hospital Clínic, Universitat de Barcelona, IDIBAPS, Barcelona, Spain
}

Background. Schizo-affective disorder has not been studied to any significant extent using functional imaging. The aim of this study was to examine patterns of brain activation and deactivation in patients meeting strict diagnostic criteria for the disorder.

\begin{abstract}
Method. Thirty-two patients meeting Research Diagnostic Criteria (RDC) for schizo-affective disorder (16 schizomanic and 16 schizodepressive) and 32 matched healthy controls underwent functional magnetic resonance imaging (fMRI) during performance of the n-back task. Linear models were used to obtain maps of activations and deactivations in the groups.

Results. Controls showed activation in a network of frontal and other areas and also deactivation in the medial frontal cortex, the precuneus and the parietal cortex. Schizo-affective patients activated significantly less in prefrontal, parietal and temporal regions than the controls, and also showed failure of deactivation in the medial frontal cortex. When task performance was controlled for, the reduced activation in the dorsolateral prefrontal cortex (DLPFC) and the failure of deactivation of the medial frontal cortex remained significant.
\end{abstract}

Conclusions. Schizo-affective disorder shows a similar pattern of reduced frontal activation to schizophrenia. The disorder is also characterized by failure of deactivation suggestive of default mode network dysfunction.

Received 23 August 2011; Revised 28 March 2012; Accepted 5 April 2012; First published online 15 May 2012

Key words: Default mode network, DLPFC, fMRI, n-back task, schizoaffective disorder.

\section{Introduction}

Schizo-affective disorder, as the name suggests, refers to a psychotic disorder that is characterized by both schizophrenic symptoms and those of mania and/or major depression. The two classes of symptom may occur simultaneously or at different times, although current diagnostic criteria require some temporal overlap, and affected patients tend to have an outcome intermediate between schizophrenia and bipolar disorder (for a review see McKenna, 2007). The nosological status of schizo-affective disorder remains a matter of controversy, with arguments that it represents a third independent form of psychosis (Procci, 1976), a form of bipolar disorder (Pope et al.

* Address for correspondence: B. L. Amann, M.D., Ph.D., FIDMAG Foundation, Benito Menni CASM, Dr Antoni Pujadas 38, 08830 Sant Boi de Llobregat, Spain.

(Email : benedikt.amann@gmail.com)
1980), a midpoint on a psychotic continuum (Crow, 1986), or the expression of genetic risk factors for both disorders (Bertelsen \& Gottesman, 1995). One traditional method for resolving such uncertainties, family history studies, has not provided decisive support for any of these positions: first-degree relatives of schizo-affective patients have variously been found to show elevated rates of schizophrenia, affective disorder or both illnesses (Coryell \& Zimmerman, 1988; Maier et al. 1993; Kendler et al. 1995; Laursen et al. 2005). Similarly, examples of all forms of psychotic disorder are seen among the monozygotic co-twins of patients with schizo-affective disorder (Cardno et al. 2012).

Another source of evidence potentially relevant to this question is brain imaging, but here the examination has been severely limited. Only two structural imaging studies of schizo-affective disorder have been carried out: an early computed tomography (CT) study found no differences in lateral ventricular 
volume among schizophrenic, schizo-affective and bipolar patients (Rieder et al. 1983), whereas a more recent magnetic resonance imaging (MRI) study found that both schizo-affective and bipolar patients had a decreased whole-brain volume compared to healthy controls (Getz et al. 2002). Functional imaging studies of schizophrenia have sometimes included some patients meeting diagnostic criteria for schizo-affective disorder; typically, however, the two groups are not separated in the analysis. It is unknown, therefore, whether patients with schizo-affective disorder show the hypofrontality that characterizes schizophrenia at rest and during task activation (Hill et al. 2004; Minzenberg et al. 2009), but which has been found less consistently in bipolar disorder, at least in the dorsolateral prefrontal cortex (DLPFC; Haldane \& Frangou, 2004; Chen et al. 2011). Nor is it known whether schizo-affective patients show the prefrontal hyperactivation that has been documented in schizophrenia during performance of working memory (Glahn et al. 2005) and other cognitive tasks (Minzenberg et al. 2009).

A further functional imaging finding in schizophrenia is failure of deactivation. This has been found in the medial frontal cortex (Pomarol-Clotet et al. 2008; Whitfield-Gabrieli et al. 2009; Milanovic et al. 2011), sometimes along with failure of deactivation in the posterior cingulate cortex (Salgado-Pineda et al. 2011; Schneider et al. 2011). Because these regions form two main nodes of the default mode network, which is a series of interconnected brain regions that are metabolically active at rest but whose activity reduces during performance of a wide range of cognitive tasks (Gusnard \& Raichle, 2001; Raichle et al. 2001), this finding has been interpreted as indicating default mode network dysfunction in schizophrenia. According to a currently limited amount of evidence, default mode network dysfunction also characterizes bipolar disorder, in terms of both failure of deactivation and abnormal resting state connectivity (Calhoun et al. 2008; Ongur et al. 2010; Pomarol-Clotet et al. 2011). Only one study has examined default mode network function in schizo-affective disorder (Ongur et al. 2010); this found reduced resting state connectivity in the medial frontal cortex in seven schizo-affective patients, which was similar in degree to that seen in seven schizophrenic patients but greater than in 17 patients with bipolar disorder.

The aim of the current study was to remedy the lack of functional imaging data in schizo-affective disorder. We examined activation patterns during performance of one of the most widely used tasks in functional imaging studies of schizophrenia, the n-back working memory task. We also examined task-related deactivations, which have been reliably demonstrated using the n-back task. Finally, we compared schizo-affective patients in manic and depressive phases of the disorder.

\section{Method}

\section{Subjects}

The patient sample consisted of 32 patients recruited from three Spanish psychiatric hospitals: Hospital Benito Menni in Sant Boi de Llobregat, Hospital Clínic of Barcelona and General Hospital of Granollers. Patients were required to meet Research Diagnostic Criteria (RDC; Spitzer et al. 1978) for schizo-affective disorder based on psychiatrist interview and review of case-notes. We used these criteria because they are the most detailed of all available criteria for schizoaffective disorder and require not only that patients show schizophrenic symptoms but also that the affective symptoms meet requirements for a full affective syndrome similar to those demanded for depression and mania/hypomania in DSM-IV and ICD-10.

Exclusion criteria included age $<18$ or $>65$ years, history of neurological disease or brain trauma, and alcohol/substance abuse within 12 months prior to participation. All of the schizomanic patients were taking antipsychotic medication (mean daily dose of chlorpromazine equivalents $=801 \pm 399 \mathrm{mg})$ and 12 patients were taking mood stabilizers (valproate, $n=9$; lithium, $n=1$; combination, $n=2$ ). One patient was on antidepressant treatment. In the schizodepressive group 12 patients were taking antipsychotic medication (mean daily dose of chlorpromazine equivalents $=475 \pm 483 \mathrm{mg}$ ), eight were on mood stabilizers (valproate, $n=2$; lithium, $n=3$; combination, $n=3$ ) and eight were taking antidepressants.

The patients were scanned while in an acute schizomanic or schizodepressive episode. Schizomanic patients were required to have a Young Mania Rating Scale (YMRS) score $>18$ and a Hamilton Rating Scale for Depression (HAMD) score $<8$. Similarly, schizodepressive patients were required to have a HAMD score $>18$ and a YMRS score $<8$.

Pre-morbid IQ was estimated using the Word Accentuation Test (Test de Acentuación de Palabras, TAP; Del Ser et al. 1997), a word reading test that requires pronunciation of Spanish words whose accents have been removed. Current IQ was measured using four subtests of the Wechsler Adult Intelligence Scale III (WAIS-III) : vocabulary, similarities, block design, and matrix reasoning. In the patients, clinical ratings included the Positive and Negative Syndrome Scale (PANSS), the Global Assessment of Functioning (GAF) and the Clinical Global Impression (CGI). 
The control sample consisted of 32 healthy individuals recruited from non-medical members of hospital staff, their acquaintances and independent sources in the community. They met the same exclusion criteria as the patients and were also excluded if they reported a history of mental illness and/or treatment with psychotropic medication.

All subjects were right-handed. The study was approved by the local ethical committee and all participants gave written informed consent.

\section{Procedure}

The participants were scanned while they performed a sequential-letter version of the n-back task (Gevins \& Cutillo, 1993). Two levels of memory load (1-back and 2-back) were presented in a blocked design manner. Each block consisted of 24 letters that were shown every $2 \mathrm{~s}$ ( $1 \mathrm{~s}$ on, $1 \mathrm{~s}$ off) and all blocks contained five repetitions (1-back and 2-back depending on the block) located randomly within the blocks. Individuals had to indicate repetitions by pressing a button. Four 1-back and four 2-back blocks were presented in an interleaved way, and between them a baseline stimulus (an asterisk flashing with the same frequency as the letters) was presented for $16 \mathrm{~s}$ to identify which task had to be performed; characters were shown in green in 1-back blocks and in red in the 2-back blocks. All participants first went through a training session outside the scanner.

\section{Behavioural data analysis}

$\mathrm{N}$-back performance was measured using the signal detection theory index of sensitivity, $d^{\prime}$ (Swets et al. 1978). Higher values of $d^{\prime}$ indicate better ability to discriminate between targets and distractors or nontargets. Subjects with negative $d^{\prime}$ values in either or both of the 1-back and 2-back versions of the task, which suggests that they were not performing the task, were excluded from the study.

\section{Functional MRI (fMRI) data acquisition}

In each individual scanning session 266 volumes were acquired from the same 1.5-T GE Signa scanner (General Electric Medical Systems, USA). A gradient echo-planar imaging (EPI) sequence depicting the blood oxygenation level-dependent (BOLD) contrast was used. Each volume contained 16 axial planes acquired with the following parameters: repetition time $(\mathrm{TR})=2000 \mathrm{~ms}$, echo time $(\mathrm{TE})=40 \mathrm{~ms}$, flip angle $=70^{\circ}$, section thickness $=7 \mathrm{~mm}$, section skip $=$ $0.7 \mathrm{~mm}$, in-plane resolution $=3 \times 3 \mathrm{~mm}$. The first 10 volumes were discarded to avoid $\mathrm{T} 1$ saturation effects.

\section{fMRI data analysis}

fMRI image analyses were performed with the FEAT module, included in FSL software (Smith et al. 2004). The following pre-statistics processing was applied: motion correction, non-brain removal, 5-mm Gaussian smoothing, grand-mean intensity normalization, and high-pass temporal filtering. To minimize unwanted movement-related effects, scans with an estimated maximum absolute movement $>3.0 \mathrm{~mm}$ or an average absolute movement $>0.3 \mathrm{~mm}$ were excluded from the study.

General linear models (GLMs) were fitted to generate individual activation maps for the contrasts: baseline versus 1-back and baseline versus 2-back. After registration to a common stereotaxic space [Montreal Neurological Institute (MNI) template], group comparisons between patients and controls were performed by mixed-effects GLM models (Beckmann et al. 2006).

We additionally examined the effect of increasing working memory load on the differences between patients and controls. To do this we fitted models that assume a linear relationship through the baseline, 1-back and 2-back levels of the task, reporting significant differences on regression slopes between the two groups.

Statistical tests were performed at the cluster level with a corrected $p$ value of 0.05 , using Gaussian random field methods. The default threshold of $z=2.3$ was used to define the initial set of clusters.

\section{Results}

\section{Demographic data}

Demographic, psychopathological and neuropsychological data for the schizo-affective patients and controls are shown in Table 1.

\section{Task performance}

The patients performed more poorly than the controls on both the 1-back $\left(d^{\prime}=3.4 \pm 1.2\right.$ v. $d^{\prime}=$ $4.4 \pm 0.7, t=3.89, p<0.001)$ and the 2-back $\left(d^{\prime}=\right.$ $2.1 \pm 1.03 v . d^{\prime}=3.2 \pm 0.9, t=4.33, p<0.001$ ) versions of the n-back task.

\section{fMRI findings}

Findings were generally more marked on the 2-back versus baseline contrast than the 1-back versus baseline contrast. However, plots of key regions were analyzed in their levels of activation/deactivation across baseline, 1-back and 2-back. Effects at the 1-back level are 
Table 1. Demographic characteristics of patients $(n=32)$ and controls $(n=32)$

\begin{tabular}{lcccc}
\hline & $\begin{array}{l}\text { Controls } \\
(n=32)\end{array}$ & $\begin{array}{l}\text { Schizo-affective } \\
\text { patients }(n=32)\end{array}$ & $\begin{array}{l}\text { Schizomanic } \\
\text { patients }(n=16)\end{array}$ & $\begin{array}{l}\text { Schizodepressed } \\
\text { patients }(n=16)\end{array}$ \\
\hline Age (years) & $44 \pm 10$ & $44 \pm 7$ & $41 \pm 8$ & $46 \pm 6$ \\
Sex (male/female) & $20 / 12$ & $20 / 12$ & $12 / 4$ & $8 / 8$ \\
TAP (pre-morbid IQ) & $22.5 \pm 4.8$ & $22 \pm 5.2$ & $23.5 \pm 5.2$ & $20.3 \pm 5.2^{\mathrm{a}}$ \\
Current IQ (WAIS-III) & $105.47 \pm 11.18^{\mathrm{b}}$ & $95 \pm 13.8$ & $100.73 \pm 16.49^{\mathrm{a}}$ & $90.56 \pm 7.4$ \\
YMRS total score & - & $13 \pm 10$ & $22 \pm 3$ & $3 \pm 3^{\mathrm{a}}$ \\
HAMD total score & - & $16 \pm 12$ & $4 \pm 4$ & $27 \pm 5$ \\
PANSS total score & - & $70 \pm 16$ & $62 \pm 13$ & $78 \pm 16$ \\
No. of affective episodes & - & $20 \pm 9.27$ & $17 \pm 12^{\mathrm{a}}$ & $21 \pm 27^{\mathrm{a}}$ \\
Illness duration (years) & - & $47 \pm 10$ & $52 \pm 9$ & $22 \pm 8$ \\
GAF score & - & $5 \pm 0.93$ & $5 \pm 1$ & $41.8 \pm 10.14$ \\
CGI score & - & & $5 \pm 0.99$ \\
\hline
\end{tabular}

TAP, Word Accentuation Test (Test de Accentuación de Palabras); WAIS-III, Wechsler Adult Intelligence Scale; YMRS, Young Mania Rating Scale; HAMD, Hamilton Depression Rating Scale; PANSS, Positive and Negative Syndrome Scale; GAF, Global Assessment of Functioning; CGI, Clinical Global Impression.

${ }^{a}$ Missing data for one patient.

${ }^{\mathrm{b}}$ Missing data for two patients.

Values are given as mean \pm standard deviation (range).

also taken into account in the analysis of working memory load (see below).

\section{Average within-group task-related activations and deactivations}

The healthy subjects showed significant activation compared to baseline in a wide network of areas. This included the insula bilaterally, the frontal operculum, middle frontal cortex and precentral gyrus extending to the bilateral DLPFC and the supplementary motor area. Also activated were the temporal, occipital and parietal cortices bilaterally, the bilateral basal ganglia and the thalamus.

The controls also showed regions of significant deactivation in the medial frontal cortex that were seen medially in the gyrus rectus and the anterior cingulate cortex anteriorly, and the precuneus posteriorly. Additionally, deactivation was observed to a lesser degree in bilateral clusters in the temporal poles extending to the hippocampus and the parahippocampus. The middle and superior temporal cortex, the posterior insula bilaterally and the left angular gyrus were also affected (Fig. 1a).

The schizo-affective patients showed activation in similar regions to the healthy subjects but this was generally less marked. They also showed deactivation in the anterior medial frontal cortex and the posterior cingulate cortex/precuneus, with the former cluster being markedly smaller than in the controls. Deactivation was also seen in the left angular gyrus. No deactivation was seen in the middle and superior temporal cortex, insula, hippocampus or parahippocampus (Fig. 1b).

\section{Differences between schizo-affective patients and controls}

The schizo-affective patients showed three clusters of significantly reduced activation compared to the controls. The largest cluster was located bilaterally $(\mathrm{L}>\mathrm{R})$ in the parietal cortex and precuneus [2620 voxels, peak activation in Brodmann area (BA) 7, MNI $(24,-68$, 52), $z$ score $=4.18, p=1.41 \times 10^{-5}$ ]. A second cluster was in the left middle frontal cortex, extending to the left precentral gyrus and reaching the left DLPFC and the left supplementary motor area [2357 voxels, peak activation in BA $44 / 6$, MNI $(32,4,54), z$ score $=4.56$, $\left.p=3.86 \times 10^{-5}\right]$. The third cluster was in the left middle and inferior temporal cortex [924 voxels, peak activation in BA 22, MNI $(-58,-48,14), z$ score $=3.71$, $p=0.02]$. These findings are shown in Fig. 2 .

The schizo-affective patients also showed significant failure of deactivation compared to the controls. This was in a cluster that included the gyrus rectus and the anterior cingulate cortex extending to the medial and superior medial frontal cortex [6619 voxels, peak activation in BA 11, MNI $(-2,40,-6), z$ score $\left.=5.01, p=4.77 \times 10^{-11}\right]$. This is also shown in Fig. 2.

Figure 3 demonstrates the plots of key regions showing levels of activation/deactivation across baseline, 1-back and 2-back. As mentioned earlier, findings were generally more marked on the 2-back 
(a)

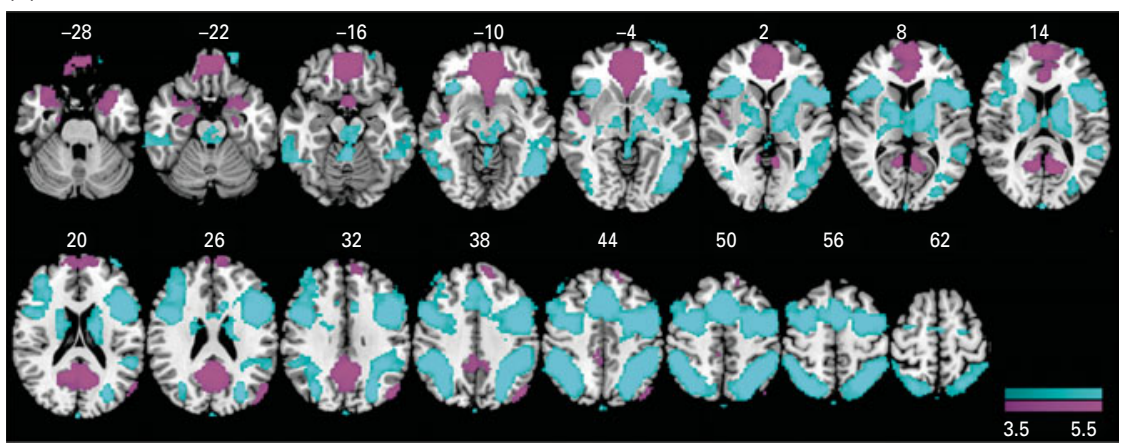

(b)

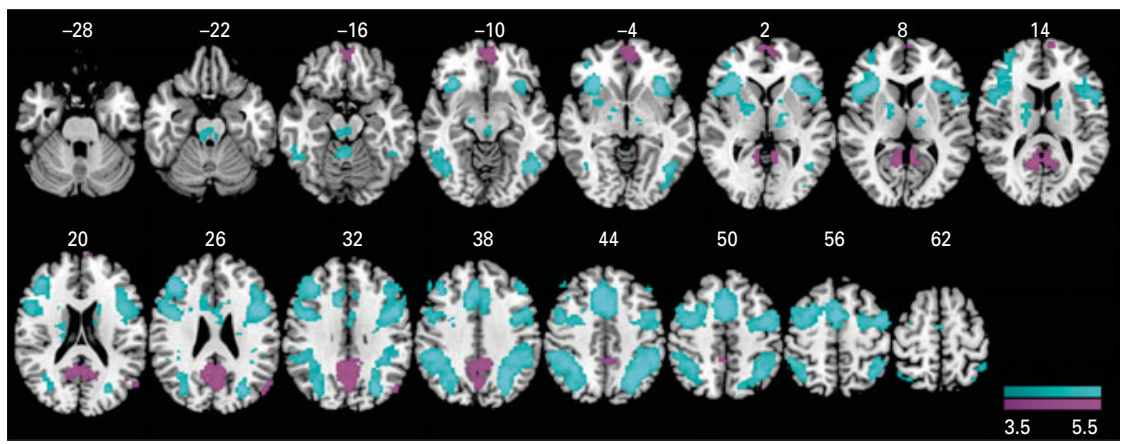

Fig. 1. Brain regions showing a significant effect in the 2-back versus baseline contrast in $(a) 32$ controls and (b) 32 schizo-affective patients. Blue indicates a positive association (activation) with the task. Pink indicates areas where the task led to a decrease in the blood oxygenation level-dependent (BOLD) response (i.e. deactivation). Numbers refer to Montreal Neurological Institute (MNI) $\mathrm{z}$ coordinates of the slice shown. The right side of each image represents the left side of the brain. Colour bars indicate $z$ scores from the group-level analysis.

versus baseline contrast than in 1-back versus baseline contrast. There were no statistical differences between the three groups on the 1-back contrast but, as shown in Figs 2 and 3, there were statistical differences on all 2-back contrasts, including a significant difference in the failure to deactivate in the anterior cingulate cortex between the two patient groups.

\section{Comparison of patients and controls by working memory load}

This analysis had broadly similar findings to the 2-back versus baseline comparison. The schizoaffective patients showed clusters of significantly reduced activation compared to the controls in three clusters. The largest cluster was in the left middle frontal cortex, extending to the left precentral gyrus, and reaching the left DLPFC and the left supplementary motor area. This cluster also extended to the left insula, the left putamen and pallidum and the thalamus bilaterally [3845 voxels, peak activation in BA 44/ 6 , MNI $(-32,-4,54), z$ score $\left.=4.78, p=4.17 \times 10^{-7}\right]$. A second cluster was located bilaterally $(L>R)$ in the parietal cortex and precuneus [3196 voxels, peak activation in BA 7 , MNI $(-34,-60,50), z$ score $=4.3$, $\left.p=3.5 \times 10^{-6}\right]$. The third cluster was located in the left middle and inferior temporal cortex [917 voxels, peak activation in BA 22, MNI $(-56,-48,16), z$ score $=3.8$, $p=0.03]$.

In this analysis the patients again showed significant failure of deactivation in a cluster in the medial frontal cortex that included the gyrus rectus and the anterior cingulate cortex and extended to the medial and superior medial frontal cortex [6296 voxels, peak activation in BA 11, MNI $(-2,40,-6), z$ score $=5.01$, $\left.p=3.6 \times 10^{-10}\right]$.

\section{Functional imaging findings in relation to task performance}

To examine the extent to which the pattern of functional imaging differences between the patients and the controls was influenced by the difference they showed in task performance, we entered each subject's $d^{\prime}$ score as a covariate in the 2-back versus baseline contrast. Specifically, we mean-centred the behavioural covariate $\left(d^{\prime}\right)$ and entered it into the linear model. After doing this, the differences in activation between the schizo-affective patients and controls persisted but became smaller in the left frontal cluster 
(a)

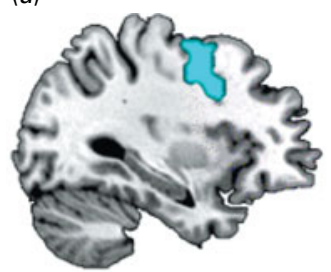

Sagittal plane

MNI $x=-32$

(b)

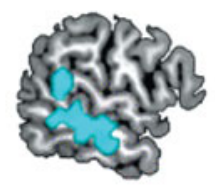

Sagittal plane

MNI $x=-58$

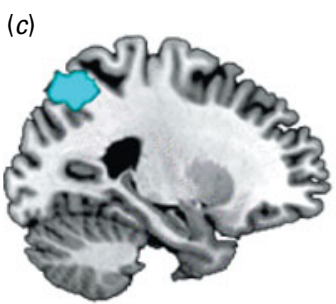

Sagittal plane

MNI $x=-24$

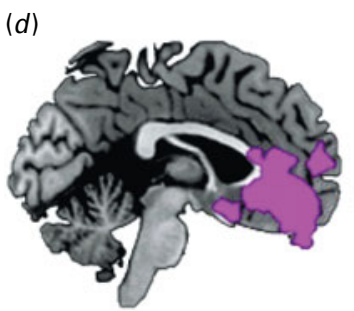

Sagittal plane

MNI $x=-2$

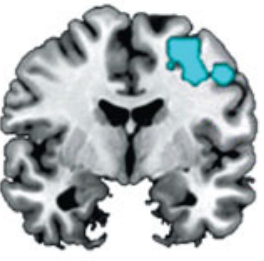

\section{Coronal plane} MNI y $=-4$

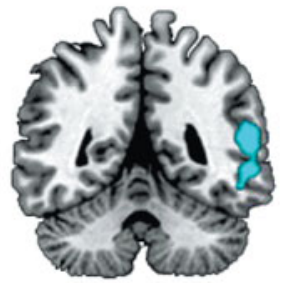

Coronal plane MNI y $=-48$

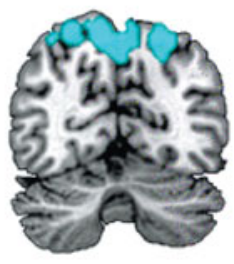

Coronal plane MNI y $=-68$

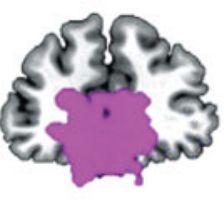

Coronal plane $\mathrm{MNI} y=40$

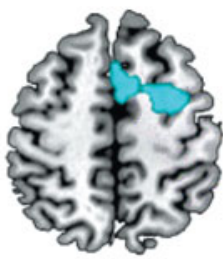

Transverse plane MNI z $=54$

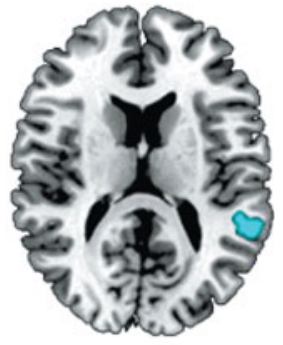

Transverse plane MNI z = 14
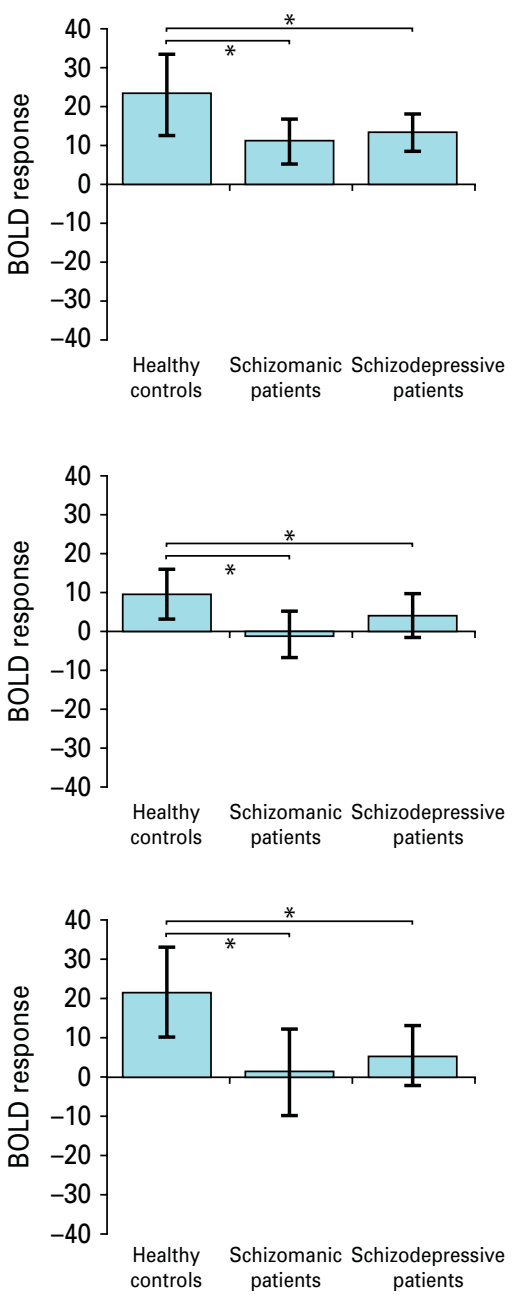

Transverse plane MNI z = 52

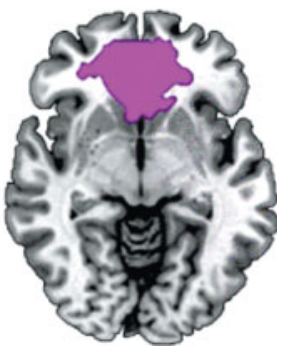

Transverse plane $\mathrm{MNI} z=-6$

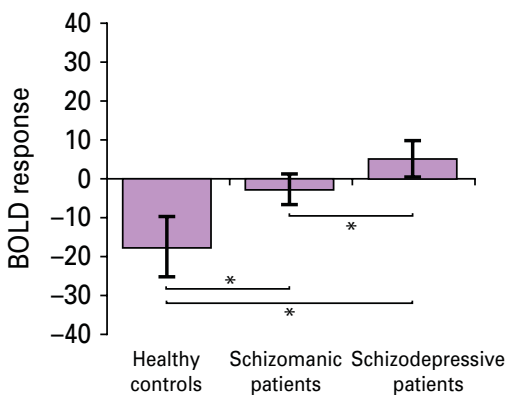

Fig. 2. Location of the (left) clusters and (right) blood oxygenation level-dependent (BOLD) response in healthy subjects $(n=32)$, schizomanic patients $(n=16)$ and schizodepressive patients $(n=16)$ of: $(a)$ the left precentral activation, $(b)$ the left middle temporal activation, $(c)$ the bilateral parietal activation and $(d)$ the anterior cingulate deactivation. The right side of the maps represents the left side of the brain.

(1529 voxels versus 2357 voxels). The clusters of reduced activation in the bilateral parietal and temporal cortex disappeared. The medial frontal cluster, where there was failure of deactivation, remained evident after covarying for task performance, although it became smaller (4290 voxels versus 6619 voxels).

\section{Relationship to clinical symptoms}

For this, we correlated the mean BOLD response from each of the above clusters of significant differences and the following clinical variables: duration of illness, number of affective episodes, PANSS score and the 
(a) Left precentral activation

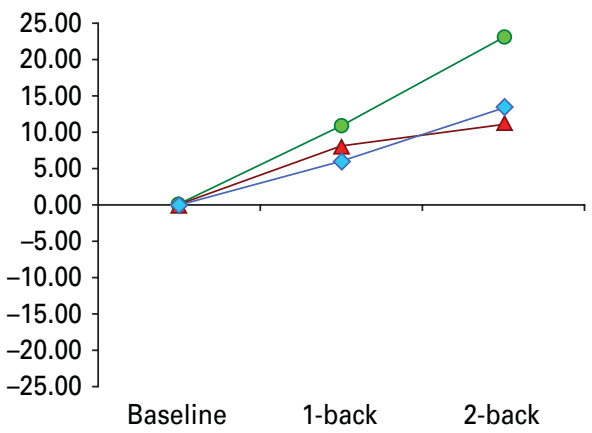

(c) Bilateral parietal activation

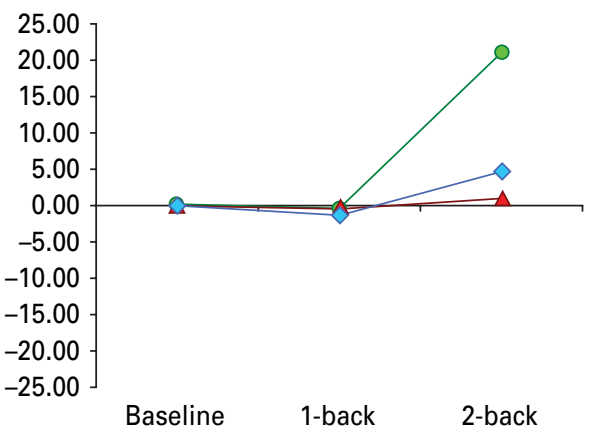

- Healthy controls $\triangleleft$ Manic $\prec$ Depressed

(b) Left middle temporal activation

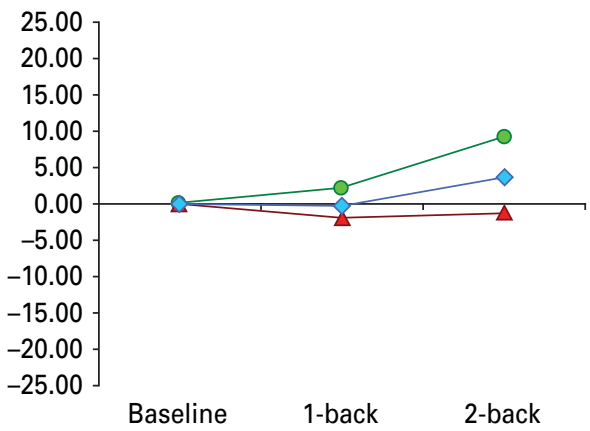

(d) Anterior cingulate deactivation

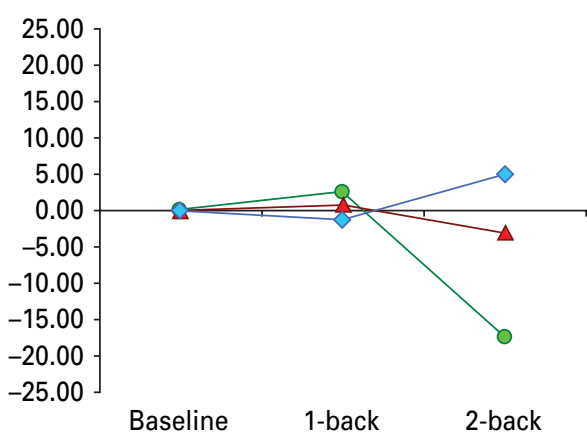

Fig. 3. Plots of $(a)$ left precentral, $(b)$ left middle temporal and $(c)$ bilateral parietal levels of activation and $(d)$ anterior cingulate deactivation across baseline, 1-back and 2-back of 16 schizomanic and 16 schizodepressive patients compared to 32 healthy controls. No differences were found in the three groups on the 1-back contrast; on all 2-back contrasts differences were statistically significant, including one between patient groups in region $d$.

two measures of overall severity of illness, GAF score and CGI score. No significant correlations were found between the left precentral, left middle temporal and bilateral parietal cortex and any clinical variable. There was a significant positive correlation between CGI score and the anterior cingulate cortex (Spearman's $p=0.45$, corrected $p=0.04$ ). However, the correlation with the other measure of severity of illness used, the GAF, was not significant.

\section{fMRI differences between schizomanic patients, schizodepressive patients and controls}

To examine these differences, the 16 schizomanic and 16 schizodepressed patients were compared in a similar whole-brain analysis to that used to compare the patients and controls (i.e. cluster threshold $z=2.3$, cluster corrected at $p=0.05)$. However, because of differences in sex, the variable sex was entered as covariates in the analysis.

There were no differences between the schizomanic and schizodepressive patients in the 1-back versus baseline contrast. In the 2-back versus baseline contrast, the schizomanic patients showed two clusters of significantly reduced activation compared to the schizodepressive. As shown in Fig. 4, one of these was a large cluster that included the anterior cingulate gyrus, the bilateral caudate and the left putamen and pallidum, the left amygdala, the left hippocampus and the left posterior part of the insula extending to the superior temporal cortex [1233 voxels, peak activation in MNI $(-52,0,-4), z$ score $=3.77, p=0.004]$. The second cluster was located in the left postcentral, precentral and supramarginal cortex and the left rolandic operculum extending to the anterior part of the insula [771 voxels, peak activation in MNI $(-58,-12,18), z$ score $=3.6, p=0.05]$.

\section{Discussion}

This is, to our knowledge, the first study to examine brain activations and deactivations in schizo-affective disorder. We examined patients who were diagnosed according to RDC, which are broadly similar to DSMIV criteria but are more detailed in their requirements, in that the affective symptoms constitute a full affective syndrome and they exclude depression secondary to delusions and hallucinations. They are also more 


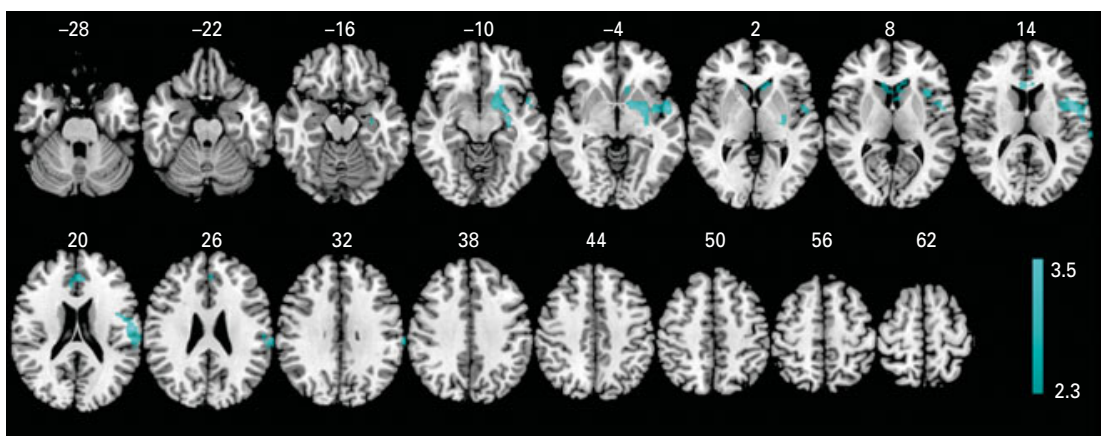

Fig. 4. In the 2-back versus baseline contrast 16 schizomanic patients showed two clusters of significantly reduced activation compared to 16 schizodepressive patients. Numbers refer to Montreal Neurological Institute (MNI) z coordinates of the slice shown. The right side of each image represents the left side of the brain. Colour bars indicate $z$ scores from the group-level analysis.

explicit about the temporal overlap with schizophrenic symptoms. We found that the patients showed reduced activation during performance of a working memory task, which affected the left DLPFC among other regions. They also showed failure of deactivation in a large area centred on the medial frontal cortex.

During the n-back performance, the healthy controls in this study showed activation in the frontal, parietal and temporal cortex, areas that show a substantial overlap with the 'working memory network' identified in a meta-analysis of fMRI studies in normal subjects (Owen et al. 2005). Activation was found to be significantly lower in the schizo-affective patients in two parts of this network, the left middle frontal cortex including the DLPFC and the parietal cortex, and also in one area outside it, the left middle and inferior temporal cortex. The finding of reduced prefrontal activation aligns schizo-affective disorder with schizophrenia, where reduced DLPFC activation is supported by meta-analyses of studies using the nback task (Glahn et al. 2005) and a wider range of executive tasks (Minzenberg et al. 2009). At first sight, our finding of reduced parietal activation does not support a proposal made by Gruber et al. (2006), who found that schizo-affective, but not schizophrenic, patients showed normal behavioural performance on tasks involving the articulatory loop component of working memory, and argued that activation in the premotor-parietal regions in the left hemisphere should therefore be preserved in patients with the former disorder. However, it should be noted that the parietal and temporal differences we found between the patients and controls disappeared when task performance was entered as a covariate in the analysis whereas those in the DLPFC remained, suggesting that dysfunction in this part of the working memory network may still distinguish schizophrenia and schizo-affective disorder. Further studies directly comparing schizophrenic and schizo-affective patients are necessary to confirm this.

Hypofrontality has been a more contentious finding in bipolar disorder. Early studies using the regionof-interest (ROI) approach (reviewed by Haldane \& Frangou, 2004) had findings of reduced, normal and increased prefrontal activation during cognitive task performance; when changes were found, they were seen in different subregions of the prefrontal cortex and showed no obvious pattern in relation to phase of illness. More recently, Chen et al. (2011) meta-analysed 50 whole-brain, voxel-based studies that examined bipolar patients using both cognitive and emotional tasks. They found consistent evidence for reduced prefrontal activation only in the inferior frontal gyrus, which was present irrespective of mood state. However, it is important to note that only one of the studies included used a working memory task. Our group has recently examined 29 bipolar manic patients using the n-back task (Pomarol-Clotet et al. 2011). Similar to the schizo-affective patients in the present study, they showed reduced activation in the DLPFC bilaterally compared to controls that extended to the precentral and supplementary motor areas; reduced activation was also seen in the right parietal cortex, including the precuneus. Bipolar depressed patients have also been found to show reduced DLPFC activation in studies using the n-back task (Townsend et al. 2010; Fernández-Corcuera et al. 2012).

In addition to reduced activation, the schizo-affective patients in our study showed failure of deactivation in an area located principally in the medial frontal cortex. Like the reduced activation in the DLPFC, this also survived controlling for performance differences between the patients and controls. Failure of deactivation, affecting a similar region and sometimes more posterior areas of the cingulate cortex, is now a replicated finding in schizophrenia (PomarolClotet et al. 2008; Whitfield-Gabrieli et al. 2009; 
Milanovic et al. 2011; Salgado-Pineda et al. 2011; Schneider et al. 2011). By contrast, studies reporting deactivations in bipolar disorder are few: our group has found failure of deactivation during n-back performance in a medial frontal/anterior temporal area very similar to that seen in the present study, which was present in both mania (Pomarol-Clotet et al. 2011) and bipolar depression (Fernández-Corcuera et al. 2012). Rubinsztein et al. (2001) also had findings consistent with failure of deactivation in the medial frontal cortex, along with the superior and middle temporal gyrus, in a study that compared manic patients and controls during performance of a gambling task.

Such findings suggest that the medial frontal cortex may be a locus of shared abnormality across major psychotic disorders. This region is of considerable topical interest because, along with the posterior cingulate cortex/precuneus, it forms one of the two important 'nodes' or 'hubs' of the default mode network. Activity in the default mode network is thought to underlie introspective or self-related thought, such as recall of personal experiences, making social and emotional judgements, envisioning the future and performing theory of mind tasks (Buckner et al. 2008). Current evidence suggests that default mode network dysfunction is a feature of a range of psychiatric and neuropsychiatric disorders including not only schizophrenia and bipolar disorder but also major depression, autism, attention deficit disorder and mild cognitive impairment (for a review see Broyd et al. 2009).

Comparing manic and depressed schizo-affective patients, both at the whole-brain level and using ROIs derived from the difference between the whole group of patients and controls, revealed differences in the medial frontal cortex. This finding should be interpreted with caution because, in the whole-brain analysis, medial frontal cortex differences were seen as part of a cluster that also included the insula and superior temporal cortex along with the basal ganglia, amygdala and hippocampus. However, it could suggest that the default mode dysfunction varies according to mood state in schizo-affective disorder. The meaning of this finding is uncertain but, given the role of the default mode network in introspection, it is tempting to speculate that it might be related to greater ruminative mental activity provoked by depressive and perhaps psychotic symptoms.

In conclusion, this study suggests that schizo-affective disorder is similar to schizophrenia in terms of being associated with hypofrontality during cognitive task performance but that it may differ from bipolar disorder in this respect (the evidence for hypofrontality not being decisive in this disorder).
However, further studies directly comparing schizoaffective patients with those with schizophrenia and bipolar disorder are necessary before firm conclusions can be drawn on this point. The study also adds to evidence that the medial frontal cortex, and by extension default mode network dysfunction, plays a pivotal, albeit non-specific, role in several forms of major mental disorder. To gain more direct insight into this issue, studies directly comparing schizo-affective and schizophrenia and/or bipolar patients are urgently needed. In common with most functional imaging studies of psychosis, the patients in this study were medicated, which could have affected activation/deactivation patterns, and this limits the conclusions that can be drawn.

\section{Acknowledgements}

This work was supported by the Centro de Investigación Biomédica en Red de Salud Mental (CIBERSAM), the Catalonian Government (2009SGR211 to the Research Unit of Benito Menni) and the Instituto de Salud Carlos: Miguel Servet Research Contracts to B. Amann (CP06/0359), R. Salvador (CP07/00048) and E. Pomarol-Clotet (CP10/00596); an intensification grant to S. Sarró $(10 / 231)$; and Research Projects to B. Amann (PI07/1278 and PI10/02622), E. PomarolClotet (PI10/01058) and R. Salvador (PI05/1874).

\section{Declaration of Interest}

None

\section{References}

Beckmann CF, Jenkinson M, Woolrich MW, Behrens TE, Flitney DE, Devlin JT, Smith SM (2006). Applying FSL to the FIAC data: model-based and model-free analysis of voice and sentence repetition priming. Human Brain Mapping 27, 380-391.

Bertelsen A, Gottesman II (1995). Schizoaffective psychoses: genetical clues to classification. American Journal of Medical Genetics 60, 7-11.

Broyd SJ, Demanuele C, Debener S, Helps SK, James CJ, Sonuga-Barke EJ (2009). Default-mode brain dysfunction in mental disorders: a systematic review. Neuroscience and Biobehavioral Reviews 33, 279-296.

Buckner RL, Andrews-Hanna JR, Schacter DL (2008). The brain's default network: anatomy, function, and relevance to disease. Annals of the New York Academy of Sciences 1124, $1-38$.

Calhoun VD, Maciejewski PK, Pearlson GD, Kiehl KA (2008). Temporal lobe and 'default' hemodynamic brain modes discriminate between schizophrenia and bipolar disorder. Human Brain Mapping 29, 1265-1275. 
Cardno AG, Rijsdijk FV, West RM, Gottesman II, Craddock N, Murray RM, McGuffin P (2012). A twin study of schizoaffective-mania, schizoaffective-depression, and other psychotic syndromes. American Journal of Medical Genetics Part B: Neuropsychiatric Genetics 159B, 172-182.

Chen CH, Suckling J, Lennox BR, Ooi C, Bullmore ET (2011). A quantitative meta-analysis of fMRI studies in bipolar disorder. Bipolar Disorders 13, 1-15.

Coryell W, Zimmerman M (1988). The heritability of schizophrenia and schizoaffective disorder. A family study. Archives of General Psychiatry 45, 323-327.

Crow TJ (1986). The continuum of psychosis and its implication for the structure of the gene. British Journal of Psychiatry 149, 419-429.

Del Ser T, Gonzalez-Montalvo JI, Martinez-Espinosa S, Delgado-Villapalos C, Bermejo F (1997). Estimation of premorbid intelligence in Spanish people with the Word Accentuation Test and its application to the diagnosis of dementia. Brain and Cognition 33, 343-356.

Fernández-Corcuera P, Salvador R, Sarró S, Goikolea JM, Amann B, Moro N, Sans-Sansa B, Ortiz-Gil J, Vieta E, Monté GC, Capdevila A, McKenna PJ, Pomarol-Clotet E (2012). Bipolar depressed patients show both failure to activate and failure to de-activate during performance of a working memory task. Journal of Affective Disorders. Published online: 10 April 2012. doi: 10.1016/ j.jad.2012.04.009.

Getz GE, DelBello MP, Fleck DE, Zimmerman ME, Schwiers ML, Strakowski SM (2002). Neuroanatomic characterization of schizoaffective disorder using MRI: a pilot study. Schizophrenia Research 55, 55-59.

Gevins A, Cutillo B (1993). Spatiotemporal dynamics of component processes in human working memory. Electroencephalography and Clinical Neurophysiology 87, 128-143.

Glahn DC, Ragland JD, Abramoff A, Barrett J, Laird AR, Bearden CE, Velligan DI (2005). Beyond hypofrontality: a quantitative meta-analysis of functional neuroimaging studies of working memory in schizophrenia. Human Brain Mapping 25, 60-69.

Gruber O, Gruber E, Falkai P (2006). Articulatory rehearsal in verbal working memory: a possible neurocognitive endophenotype that differentiates between schizophrenia and schizoaffective disorder. Neuroscience Letters 405, 24-28.

Gusnard DA, Raichle ME (2001). Searching for a baseline: functional imaging and the resting human brain. Nature Reviews Neuroscience 2, 685-694.

Haldane M, Frangou S (2004). New insights help define the pathophysiology of bipolar affective disorder: neuroimaging and neuropathology findings. Progress in Neuropsychopharmacology and Biological Psychiatry 28, 943-960.

Hill K, Mann L, Laws KR, Stephenson CM, Nimmo-Smith I, McKenna PJ (2004). Hypofrontality in schizophrenia: a meta-analysis of functional imaging studies. Acta Psychiatrica Scandinavica 110, 243-256.

Kendler KS, Neale MC, Walsh D (1995). Evaluating the spectrum concept of schizophrenia in the
Roscommon Family Study. American Journal of Psychiatry $152,749-754$.

Laursen TM, Labouriau R, Licht RW, Bertelsen A, Munk-Olsen T, Mortensen PB (2005). Family history of psychiatric illness as a risk factor for schizoaffective disorder: a Danish register-based cohort study. Archives of General Psychiatry 62, 841-848.

Maier W, Lichtermann D, Minges J, Hallmayer J, Heun R, Benkert O, Levinson DF (1993). Continuity and discontinuity of affective disorders and schizophrenia. Results of a controlled family study. Archives of General Psychiatry 50, 871-883.

McKenna P (2007). Schizophrenic and Related Syndromes, 2nd edn. Routledge: Hove, UK.

Milanovic SM, Thermenos HW, Goldstein JM, Brown A, Gabrieli SW, Makris N, Tsuang MT, Buka SL, Seidman LJ (2011). Medial prefrontal cortical activation during working memory differentiates schizophrenia and bipolar psychotic patients: a pilot FMRI study. Schizophrenia Research 129, 208-210.

Minzenberg MJ, Laird AR, Thelen S, Carter CS, Glahn DC (2009). Meta-analysis of 41 functional neuroimaging studies of executive function in schizophrenia. Archives of General Psychiatry 66, 811-822.

Ongur D, Lundy M, Greenhouse I, Shinn AK, Menon V, Cohen BM, Renshaw PF (2010). Default mode network abnormalities in bipolar disorder and schizophrenia. Psychiatry Research 183, 59-68.

Owen AM, McMillan KM, Laird AR, Bullmore E (2005). N-back working memory paradigm: a meta-analysis of normative functional neuroimaging studies. Human Brain Mapping 25, 46-59.

Pomarol-Clotet E, Moro N, Sarro S, Goikolea JM, Vieta E, Amann B, Fernandez-Corcuera P, Sans-Sansa B, Monte GC, Capdevila A, McKenna PJ, Salvador R (2011). Failure of de-activation in the medial frontal cortex in mania: evidence for default mode network dysfunction in the disorder. World Journal of Biological Psychiatry. Published online: 23 May 2011.

doi: 10.3109/15622975.2011.573808.

Pomarol-Clotet E, Salvador R, Sarro S, Gomar J, Vila F, Martinez A, Guerrero A, Ortiz-Gil J, Sans-Sansa B, Capdevila A, Cebamanos JM, McKenna PJ (2008). Failure to deactivate in the prefrontal cortex in schizophrenia: dysfunction of the default mode network? Psychological Medicine 38, 1185-1193.

Pope Jr. HG, Lipinski JF, Cohen BM, Axelrod DT (1980). 'Schizoaffective disorder' : an invalid diagnosis? A comparison of schizoaffective disorder, schizophrenia, and affective disorder. American Journal of Psychiatry 137, 921-927.

Procci WR (1976). Schizo-affective psychosis : fact or fiction? A survey of the literature. Archives of General Psychiatry 33, 1167-1178.

Raichle ME, MacLeod AM, Snyder AZ, Powers WJ, Gusnard DA, Shulman GL (2001). A default mode of brain function. Proceedings of the National Academy of Sciences USA 98, 676-682.

Rieder RO, Mann LS, Weinberger DR, van Kammen DP, Post RM (1983). Computed tomographic scans in 
patients with schizophrenia, schizoaffective, and bipolar affective disorder. Archives of General Psychiatry 40, 735-739.

Rubinsztein JS, Fletcher PC, Rogers RD, Ho LW, Aigbirhio FI, Paykel ES, Robbins TW, Sahakian BJ (2001). Decision-making in mania: a PET study. Brain 124, 2550-2563.

Salgado-Pineda P, Fakra E, Delaveau P, McKenna PJ, Pomarol-Clotet E, Blin O (2011). Correlated structural and functional brain abnormalities in the default mode network in schizophrenia patients. Schizophrenia Research 125, 101-109.

Schneider FC, Royer A, Grosselin A, Pellet J, Barral FG, Laurent B, Brouillet D, Lang F (2011). Modulation of the default mode network is task-dependant in chronic schizophrenia patients. Schizophrenia Research 125, 110-117. Smith SM, Jenkinson M, Woolrich MW, Beckmann CF, Behrens TE, Johansen-Berg H, Bannister PR, De Luca M, Drobnjak I, Flitney DE, Niazy RK, Saunders J, Vickers J, Zhang Y, De Stefano N, Brady JM, Matthews PM (2004). Advances in functional and structural MR image analysis and implementation as FSL. NeuroImage 23 (Suppl. 1) S208-219.

Spitzer RL, Endicott J, Robins E (1978). Research diagnostic criteria: rationale and reliability. Archives of General Psychiatry 35, 773-782.

Swets JA, Green DM, Getty DJ, Swets JB (1978). Signal detection and identification at successive stages of observation. Perception and Psychophysics 23, 275-289.

Townsend J, Bookheimer SY, Foland-Ross LC, Sugar CA, Altshuler LL (2010). fMRI abnormalities in dorsolateral prefrontal cortex during a working memory task in manic, euthymic and depressed bipolar subjects. Psychiatry Research 182, 22-29.

Whitfield-Gabrieli S, Thermenos HW, Milanovic S, Tsuang MT, Faraone SV, McCarley RW, Shenton ME, Green AI, Nieto-Castanon A, LaViolette P, Wojcik J, Gabrieli JD, Seidman LJ (2009). Hyperactivity and hyperconnectivity of the default network in schizophrenia and in first-degree relatives of persons with schizophrenia. Proceedings of the National Academy of Sciences USA 106, 1279-1284. 